\title{
Bio-inspired vertebral column, compliance and semi-passive dynamics in a lightweight humanoid robot
}

\author{
Olivier Ly $^{(1)}$, Matthieu Lapeyre ${ }^{(2)}$ and Pierre-Yves Oudeyer ${ }^{(2)}$
}

\begin{abstract}
This paper presents the humanoid robot Acroban. We study two main issues: 1) Compliance and semi-passive dynamics for locomotion of humanoid robots regarding robustness against unknown external perturbations; 2) The advantages of a bio-inspired multi-articulated vertebral column.

We combine mechatronic compliance with structural compliance due to the use of flexible materials. And we explore how these capabilities allow to enforce morphological computation in the design of robust dynamic locomotion. We also investigate the use of compliance to design semi-passive motor primitives using the torso and the arms as a system of accumulation/release of potential/kinetic energy.
\end{abstract}

keywords. Lightweight Humanoid Robot, Morphological Computation, Semi-Passive Dynamic Walking, Compliance, Vertebral Column, Spine, CPG.

\section{INTRODUCTION}

The problem of robot locomotion and in particular biped walking has facinated people for a long time, and still is a major issue of modern robotics. The challenge is to make robots move in the same manner as humans so as to fit into the human environment, and also to be socially accepted. Let us mention the famous Honda's Asimo or HRP-4.

This paper presents the humanoid robot Acroban. We study two main issues: 1) Compliance and semi-passive dynamics for dynamic locomotion of humanoid robots regarding robustness to unknown external perturbations, and 2) the advantage of a bio-inspired multi-articulated vertebral column in the dynamics of motor primitives.

Vertebral Column. Acroban has a humanoid mechanical structure (see Figure 1 and Video 1). It has 30 degrees of freedom. Its height is about $70 \mathrm{~cm}$. Its size is comparable to the NAO, the Qrio, or the Darwin ([22]) robots. Acroban has the classical joints of humanoid robots, but in addition it includes a multi-articulated compliant vertebral column with 5 degrees of freedom.

We no longer consider the trunk as a rigid undeformable body. And thus, this opens a new field of motions involving subtle changes in the mass distribution of the robot. This design provides a strong independency between the higher part of the body (shoulder, head and arms) and the lower part (legs and pelvis). This allows the legs and also the pelvis to move without implying moving the upper part of the body. This is illustrated in particular during gait, where the spine is used for horizontal lateral mass transfer, relieving hip joints.

This research project was partially funded by ERC Starting Grant EXPLORERS 240007.

(1) INRIA/LaBRI, Bordeaux I Univ.,France ly@labri.fr

(2) FLOWERS, INRIA Bordeaux, France

$\{$ matthieu.lapeyre, pierre-yves.oudeyer\}ainria.fr
In a bio-inspired spirit, the structure of Acroban's vertebral column implements several fundamental properties of the human vertebral column as emphasized in [5].

At the moment, a few robots like iCub ([18]), Wabian ([3]) or bio-inspired robots like the ECCE robot ([15]), the WBD-1 ([24]) or Cla and Rabbit ([21]) and Kenta ([19], [20]), or the Ijspeert's Salamander ([13]) have a vertebral column; most of them use it to enlarge the operational field of arms; but Acroban may be the only biped robot in this family that is able to walk and keep balance relying heavily on its articulated vertebral column.

Compliance. We consider compliance as the ability of the system to absorb and to go with external physical perturbations. Compliance is the inverse of stiffness. This means that the system can be deformed (in a large sense, including joint's mobilities) by the action of unpredicted external forces. Acroban is compliant at two levels: First, the mechatronic of Acroban, while controlled in position, allows the real-time adjustment of the maximal torque that a joint is authorized to enforce. This allows to limit the resistancy of each joint against an external perturbation and in this way to make it compliant. Moreover, Acroban is able to get real-time information about the force applied to a particular compliant joint through the position error. Second, the mechanical structure itself is compliant: Acroban structure includes springs and elastics which, together with the backlash of the mechanical structure and of servomotors, makes it flexible. In top of that, soles of Acroban are covered by a compliant material.

However, Acroban does not enforce force control. Indeed, there is no real-time force enslavement. And reactions to perturbations are not producted by computations and decisions of the control system. Thus Acroban's motor behaviour would better be qualified as semi-passive. We call a system passive when it has no actuator at all, and uses physic as only controller. They have been introduced in [16]. Also, such a system uses potential energy as unique energy source; and in an analogous way, Acroban uses spring's potential energy as energy accumulator. Such systems have also been investigated e.g. in [23]. Let us emphasize the immediate nature of (semi)-passive reactive motion, which are driven directly by physic and thus acts without any delay.

Motion Control. First of all, the stabilizing system of Acroban implement an independant feedback loop system which makes the knees, the hips and the pelvis react in order to keep balance on the base of forces applied to ankles and also an accelerometer and gyroscopic sensor (see Section II-B for details). 
But the stabilizing system does not rely only on that. It also heavily relies on (semi-passive) compliance: First, the compliance of the higher part of the body (thanks to the vertebral column) acts as a system coupling a semi-passive inverse pendulum (the spine) and two passive pendulums (the two arms), (see Section III-B). The motions of these pendulums are kept by active joints (legs and pelvis). And in turn, they contributes to the lateral weight transfer, by transferring its potential energy to motion. Experiments showed that this process decreases power consumption and also the effect of inelastic shock at foot landing. Second, the ankles are made also compliant which decreases again the effect of the inelastic shocks. Finally, feets include compliant material and have partially slippery soles which makes the legs adjust the positions of the feet by themselves by slightly gliding on the ground.

Locomotion is then considered as a perturbation selfgenerated by central pattern generators (CPG for short) of the legs and of the pelvis and we use the previous system to make the robot spontaneously stabilize itself. In particular, Acroban does not involve analytically real-time computed dynamics, neither ZMP based controller. Acroban's gait actually is semi-passive, following the trend of self-stabilizing passive mechanisms opened in [16] and of powered semi-passive robots (see [7]); the mechanism of semi-passive balance of the torso of Acroban could be compared to the powered passive dynamic walker of [35].

The use of compliance as stabilizing controller as described above transforms the whole mechanical structure into a distributed system looking for local minima of potential energy and stable configurations and trajectories. It absorbs shocks (including unpredicted ones) and adjusts constantly the position of the robot without high-frequency global control

This doing, it relieves the global motion control, which is does not have to enforce centralized high-frequency reactions. In that, we follow ideas of morphological computation (see e.g. [26], [28]) by making the mechanical structure ensure a large part of the control (see [25]).

This distributed nature of motion control actually extends into a whole modular system where are mixed independant mechanical reactions as mentionned above, balancing controllers and even motions (e.g. the gait). In fact, this extends the classical position-control robotic architecture where each servomotor enforces a position control of its joint as an independant unit which not aware of the dynamic of the whole robot.

Acroban does not enforce computation of the global dynamic of the robot, and the global feedback loop has low frequency $(50 \mathrm{~Hz})$. Taking into account the low frequency of global feedback of biological organisms, we also investigate the question of how low can be the global feedback control loop of a humanoid robot.

The use of compliant robots is not mainstream in the context of locomotion. However, number of projects have already shown the importance of an adequate compliant morphology for motor skills. This is illustrated by the quadruped BigDog whose compliance relies on hydraulic actuators ([29]), see also [14]. Bio-inspired robots use also compliance (e.g. Rollin Justin Arms [10], ECCE Robot [15], robots of the PHRIENDS project [1], [4], [12]) and especially material compliance (see e.g. RHex [32] or [11], [34] [8], [17]). Besides, compliance is also essential in the design of leg prosthesis (see e.g. [38]). In these designs, one can observe that compliance greatly improves robustness of locomotion. However, the cost of this robustness is less predictability and less accuracy.

Thanks to the small size of Acroban, the experimentation process is drastically simplified regarding the ease of manipulation and security. The ratio material resistance / weight implies a better robustness of the mechanical structure, making Acroban able to fall without breaking.

This changes deeply the process of motor skill design by allowing creating motions directly on the robot by direct experiments without needing any simulation process. This includes for instance adjusting in real-time motor primitives, and even extreme ones like stabilizing process (see Video 4).

However, at the same time, the small size makes difficult the integration of highly accurate mechatronic components. Backlash together with the use of springs and the relative inaccuracy of the structure, implies that analytic models of the platform are difficult to exploit. This makes the easiness of the experiment process particularly important. It is also important seeing that our focus for future research is motor learning, and especially locomotion.

This article comes with accompanying videos available on Youtube, as indicated in the following (also available on http: / / www - youtube. com/user/Inriaflowers).

Video 1: General architecture of Acroban

http: / / www . youtube.com/watch? v=zHbl-ozA_ho

Video 2: Dynamically controlled mechatronic compliance

http: / / www . youtube. com/wat ch? v=0g72 SdI JcaM

Video 3: Intrinsic Structural Compliance

http: / / www . youtube. com/wat ch?v=p3aUa5FGL $4 \mathrm{~s}$

Video 4: Stabilization and robustness to unknown external perturbations

http: / / www . youtube. com/watch?v=EENWCI0OIYC

Video 5: Illustrating passive dynamic walking in Acroban

http: / / www . youtube. com/wat ch? v=gKEjkckxzBU

Video 6: Robust semi-passive dynamic walking

http: / / www . youtube. com/watch? v=EKLl4Z1weHo

\section{ARCHITECTURE}

\section{A. Mechanical Structure}

1) Global Structure: The Acroban platform is a small (about $70 \mathrm{~cm}$ ) and lightweight (about $5 \mathrm{~kg}$ ) humanoid robot with many degrees of freedom (30 dofs) and a multiarticulated spine. The structure only includes revolute joints, which are all actuated by servomotors (see Section II-A) in a modular way: Each ankle has 3 joints enforcing a spherical link. Each knee has 1 joint enforcing a revolute link. Each hip has 3 joints enforcing a spherical link. The vertebral column has 5 joints. Each shoulder has 1 joint enforcing a 2-revolute joints link. Each elbow has 2 joints enforcing a 
2-revolute joints link. We essentially focused on designing a mechanically rich and open structure in the area of the vertebral column and the pelvis, providing it with 11 degrees of freedom.

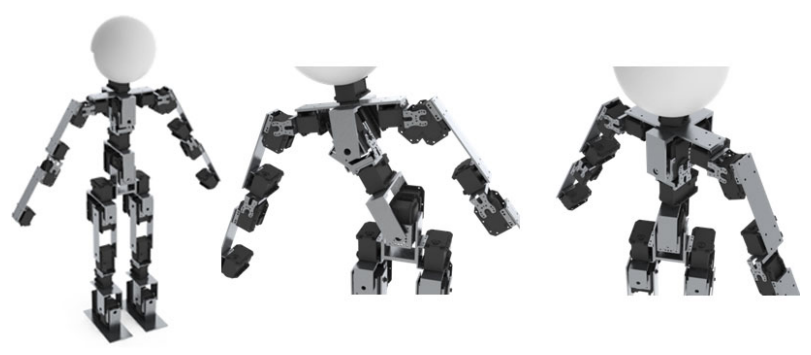

Fig. 1. Acroban Global Structure

2) Vertebral column: The vertebral column can be viewed as a system linking the pelvis and the shoulder. It enforces two revolutes joints links at its two extremities, each of them providing rotations in the sagittal and the coronal planes and one in the transverse plane. During motions, and in particular motions related to locomotion, this allows getting independency of the higher part and the lower part of the body. This allows for instance to reduce the dynamic of the higher part of the body during the gait. We claim that this contributes significantly to the stabilisation of the robot.

3) Pelvis: The pelvis, seen as an independent sub-body, may have several kind of mobility. It produces precise movements of the center of gravity of the robot. Firstly, it can move by a rotation in the sagittal plane. We will use it extensively to keep balance. Secondly, it can move in the transverse plane, this is used for the gait for the weight transfer between the legs, instead of making the legs support all the efforts of displacement of the body.

\section{B. A Bio-inspired Design}

The Human gait is an undeniable reference for the study of locomotion. Even if it is far from being clear that a direct transposition of human gait to robots is really effective, the mechanical and control processes generated by thousands years of evolution to solve bipedal locomotion problem are an important source of information and inspiration for humanoid robotics [2]. Many researchers were interested in biomechanics of human walking (see e.g [31]). These studies describe accurately the kinematics and dynamics of legs during walking (see [37]).

Among the large litterature concerning human biped walking, only a few projects studied the role of the trunk during walking. Yet the trunk represents $60 \%$ of the total weight for humans, which raises the center of gravity (see e.g. [36], [9]). The trunk has a large complex network of muscles used to accomplish a lot of movements while keeping the balance. Its movements are regulated by a complex combinaison of anticipatory and reactive actions. The movements of the spine can facilitate the transfer of weight from one leg to the other one, improve the balance but also participate to the dynamic of the walking. It seems therefore interesting to enable a humanoid robot trying to walk in a robust way, to have an articulated trunk. But even if the human trunk has already been designed in some robots (like ECCE Robot [15]), it is difficult to replicate on a small robot using servomotors. So we must simplify and find the most essential degrees of freedom of the spine. Interestingly, Ceccato [5] studied the role of the trunk and highlighted the main displacements of the spine during walking. And the apparent high dimensionality of the trunk appears to be factorizable down to a few essential components/dimensions. First, experiments showed small oscillations in the pelvis and the thoracic in the sagittal plane, highlighting that only two joints, one for the pelvis and one for the thoracic should be sufficient to represent the motion of the spine in the sagittal plane. In the coronal plane, the pelvis and shoulders oscillate in phase opposition while the middle remains straight throughout the cycle. This implies that essential movements of the trunk in the coronal plane could be approximated using two joints, one for the pelvis and an other one for the shoulder. Finally, in the horizontal plane, there are opposite rotations between the upper trunk and the lower trunk, enforced by a twist of the spine. So, only one revolute joint in the middle of the spine should be sufficient.

Accordingly, Acroban has five joints for the trunk, as shown on figure 1 (see also Video 1): Two in the sagittal plane and two in the coronal plane, placed in the pelvis and shoulder/thoracic and one in the horizontal plane placed in the middle of his trunk. With this design, we have the strictly minimum necessary joints to replicate essential degrees of freedom of the human trunk.

\section{Mechatronic \& COnTRol}

\section{A. Mechatronic}

Servomotors. Acroban is actuated by standard servomotors that enforce revolution joints: Dynamixel ${ }^{1} \mathrm{RX}-64$ and RX-28. RX-64 (resp. RX-28) can deploy $64 \mathrm{~kg} . \mathrm{cm}^{-1}$ (resp. $28 \mathrm{~kg} . \mathrm{cm}^{-1}$ ) in standing torque, decreased around $30 \mathrm{~kg} . \mathrm{cm}^{-1}$ $\left(15 \mathrm{~kg} . \mathrm{cm}^{-1}\right)$ during movements. They have small backlash and low accuracy due to classical metal gear reductor (not harmonic neither planetar), however their mechanic is reversible which allows to get them compliant. The servomotors enforce position control. However, they allow adjusting in real time a maximal bound for the torque produced by the joints. Moreover a driving mode called "null torque mode" in which the servomotor cancels the rubbing forces of the gears and of the motor in order to get a completely passive joint is available.

Springs. Some particular joints (knees, coronal hip and pelvis) are lightly under-powered. We address this problem by using torsion springs as energy accumulator to support servomotors and also to introduce compliance.

Elastics. The use of standard servomotors implies a significant backlash. To address this problem, we use elastics (or extension springs) set between the extremities of the leg which replaces the backlash by a kind of force proportional

\footnotetext{
${ }^{1}$ Dynamixel RX64 User's Manual: www.robotis.com (CDynamixel
} 
reaction in the backlash range. We limited the use of elastics to legs as these last ones are crucial for locomotion.

\section{B. Motor Control System}

Servomotors are controlled by a centralized upper layer implementing the logic of movements. This layer is enforced by an embedded system based on ARM technology. It schedules motions by discretizing time at low frequency: $50 \mathrm{~Hz}$. However, each servomotor embeds a control loop of higher frequency (higher than $1 \mathrm{KHz}$ ); but these control loops are independent from each other, and targeted to the position control only.

Motor Primitives. Movements are subdivided into modules called parameterized motor primitives. Motor primitives are combined in order to define motions of Acroban in a modular way. In turn, motor primitives are constructed by combinations of splines, CPGs and PID controllers which are all runned in parallel by the upper layer described above. Splines are defined by the user point by point. CPGs rely on sinusoids whose period, phase and amplitude are defined by the user. Propotional-Integral-Derivative (PID for short) controllers classically take multidimensional entry signals, and compute output by weighted sum of proportional, discrete integral and discrete derivative operation. The user adds as many PID controller that he wants, and defines output, gains, and entry signals among sensor values (accelerometer, gyroscopic) and/or position errors of the joint. Let us mention that we added basic filters to PID controllers: mininum and maximum values, and mobile discounted average whose parameters are defined by the user.

Finally, at each time and for each kind of output (see below), all the coresponding values of these modules are simply sumed up to get the global output value.

Outputs are joint position targets, joint maximal torques (and thus, as explained in Introduction, compliance), and also position in the operational space (cartesian positions of the feet computed by inverse kinematic); more complex motions are obtained also by taking as output motor primitive parameters (typically the gains of splines, or CPG, but also of PID controller).

\section{Robust Compliant Stabilizing and Dynamic Gait as a Stabilized SElf-Perturbated Motor PRIMITIVE}

Our goal consists in designing stabilizing motor primitives able to maintain the system stable under various external mostly unknown perturbations. In particular, such primitives are designed quasi-independently from the walking CPG. The gait then results from a stabilization motor primitive perturbed by an active CPG coming from the lower part of the body (pelvis and legs).

Motor Primitive Designing Method. The hybrid nature of the structure of Acroban, involving several kind of materials possibly flexible, the relative inaccuracy of the design, the backlash and the inaccuracy mechatronic implies that analytic models of the platform are difficult to exploit in order to generate motor primitives by simulation.
Motor primitives are actually defined and tuned by hand within an experimental process involving online tests (trials and errors) on the real robot. In order to get objective assessments and both quantitative and qualitative performance measures, we rely on a motion tracking device ${ }^{2}$ to analyse Acroban's motions.

The process of designing stabilizing and walking motor primitives relies on several criteria, aiming at evaluating the quality of the stabilizing process. Among them: speed of convergence to static stability, oscillation amplitude for stabilizing, periodicity, cycle stability for walking (see Section IV for details). Let us note that the most basic criterion is the number of steps without falling. Due to the lack of space, we will not give the details of final parameter values, we neither will give formal forms of PID controlers.

\section{A. Compliant stabilizing motor primitives}

We have experiment several stabilizing motor primitives. As mentionned above, all of them have been designed by experiment. In the following we describe the modules constituting these motor primitives. We describe the nature of the modules in a qualitative way; parameters are tuned by experiments and does not deserve to be outputed here. Each of them has two parts: A controller and a compliance mode.

1) Compliance: Compliance is twofold: controlled (see Video 2) and structural (see Video 3).

Controlled compliance means adjusting the maximal torque of joints during motion. In a static way where the compliance of a particular joint is fixed for the whole motion, this means that one puts the structure into a configuration with particular morphological computation properties, which can be akin to morphosis (see [6]). In a dynamic way, one typically simulate an adjustable spring (see e.g. [27]). We use this technique at different levels of the robot:

- Ankles. We make the ankles compliant in the coronal plane. This ensures a good adherence of the feet robust to perturbations. This is enforced by a simulated adjustable spring in turn enforced by a PID controller taking the position error of the ankle and acting on its maximal torque.

- Pelvis and vertebral column. We make compliant the coronal joints of the vertebral column and of the pelvis. The higher joint of the vertebral column is made completely free (null maximal torque), while the lower joint behaves like an ajustable spring.

- Arms and shoulders are also made compliant, and even free (see below).

- Sagittal rotation of the pelvis. We adjust a relative compliance of the sagittal rotation of the pelvis in order to absorb sagittal moment acting on the whole structure. Again, this is enforced by a simulated adjustable spring.

Let us note that the fact that compliance is dynamically controlled by PID controllers makes the transition between compliant/passive state and stiffness continuous.

\footnotetext{
2 Our motion tracking device records motion of an IR led in a plane parallel to the CCD. In our experiments, this Led is placed on the head of Acroban
} 
Structurally, comparing to a body which would have just legs, the upper part of the body (torso, head and arms) makes the center of mass move up. This makes higher the amplitude of movements of the center of mass in the horizontal plane, and thus makes easier the lateral weight transfer from one leg to the other one. However, at the same time it makes the system more instable. This is mitigated by the vertebral column, the shoulder and the arms, made compliant as described above, making the torso-arm system behaves like three coupled pendulums, i.e. the spine as an inverted pendulum to the top of which are attached two pendulums (the arms). Experiments show that such a system absorbs shocks and improves the stability of the system.

Structural compliance (i.e. flexibility of the structure, and in particular legs) of Acroban also plays an important role (see Video 3). It absorbs the impact of foot landing and helps the leg to get in a locally stable configuration by itself. Quantitatively, along the whole body of Acroban, and all motors stiff, the flexibility range is approximately 20 as can be seen on Video 3.

Finally, let us mention the slippery sole of Acroban. During the foot landing, it reduces the horizontal component of the impact in a very important way, avoiding important perturbations, and makes the leg find by itself local horizontal adjustment of the foot position. Experiments show that this feature improves considerably the stability of the robot during walk. However, this costs a lack of precision and of efficiency of steps, which adapt this way constantly to the environment.

2) Active Controllers: The stabilization system also relies on active controllers. Like above, we describe these controller in a qualitative way seeing that their parameters are adjusted by experiment.

- Deformable parallelogram movements on the vertebral column. In this way, one corrects the vertical position of the vertebral column while bringing only minimal moment of the higher part of the body. This motion emphasizes a significant improvement of the dynamics of the robot by the vertebral column. This action is enforced by a PID controller taking the gyroscopic sensor as entry.

- Ankle and knees positions in the sagittal plane. We use them to counter the lean of the robot via a PID controller taking the the gyroscopic sensor as entry.

- Pelvis sagittal rotation. This motion produces precise movements of the center of mass while bringing only minimal moment on the remaining of the body. We use it also to maintain the pelvis horizontal. It is enforced by two PID controllers taking as entry the accelerometer sensor and the gyroscopic sensor.

- Center of gravity. By using inverse kinematic of legs, one has a control of the higher part of the body in the Cartesian space. We use it as an approximation of Cartesian movements of the center of mass:

- Horizontal movements are used at low frequency to correct the position of the body. Low and high frequency selection is done by mobile discounted average of the entry signal (accelerometer), and a PID controler is enforced on the smoothed signal.

- Horizontal movements are used at high frequency to absorb horizontal perturbations.

- Vertical movements are used to transform the position of the robot into a minimum of potential energy, and in this way transform the position into an attractor. This is enforced by a PID controller taking as entry the smoothed accelerometer.

We use also a controller of higher level: the amplitude and the direction of steps. In the waking motor primitive, we define the step by means of splines that describe the trajectory of the foot in the Cartesian space (this trajectory is again tuned by experiment). Roughly speaking, the robot stabilizes itself by doing forward steps if he leans forward, and backward steps if he leans backward. For doing that, we use the lean of the robot (taken from the accelerometer) to determine the vertical amplitude of the step via a PID controller.

Coronal stabilisation relies essentially on compliance: The system made of the vertebral column, the shoulders and the arms is a combination of an inverted pendulum (the vertebral column) and two pendulums (the arms coupled with shoulders), actuated by the lower coronal rotation of the vertebral column, which is compliant, but with a compliance degree controlled by its position error (see Video 4).

\section{B. Passive and Semi-Passive Dynamic Gait}

1) Passive Dynamic Gait: Considering the stabilizing motor primitives described above, a particular family of external perturbations, i.e. periodic lateral perturbations here generated by a human, provokes spontaneously passive dynamic walking as shown in Video 5.

While the robot uses the same stabilizing motor primitive, this perturbation, amplified by the triple pendulum in the torso, makes it step forward as a consequence of the mechanical elasticity of the feet and legs. Indeed, during grounded phase, the leg accumulates energy for horizontal translation and torsion motions of the foot; and it frees this energy when the foot is up, making this last one move and perform a step (see video 5).

2) Dynamic Gait as a Stabilized Self-Perturbation: Starting from this intrinsic capability for passive dynamic walking, we designed a motor primitive, based on the use of a CPG directly added to the stabilizing motor primitive described above. In this way, one generates dynamic gait by a self-perturbation. In this case, we have what one may call a powered passive walker or a semi-passive walker.

The walking loop pattern has two sides: An active side driven by the legs and the pelvis, mixing lateral weight transfer and step forward. This active side is generated by CPG. A passive side consisting in one of the stabilizing motor primitive as described above.

The use of the double coupled pendulum system described above makes the robot use potential energy as local energy accumulator. The movement of the pendulum is kept by active movements of legs and pelvis. But at the same time 
it contributes significantly to the weight transfer: First it changes the global mass distribution; and second, it induces a reaction moment on the lower part of the body via the pelvis. Therefore, this movement has a semi-passive nature. Let us note that the active use of the pelvis, independently of the torso for the step is largely inspired by the human walk (see e.g. [37],[30]).

Besides, it is possible to control the whole walking pattern, i.e. to get the robot turn in all directions and walk forward at various speeds, by controlling the amplitude of its splines, independently for left and right step. This reduces the control of the walk to 2 parameters determining first the sum of the amplitudes of both steps and seconds the relative difference between them.

We also tested the same motions but with stiff joints in the vertebral column and the arms. Experiments showed that the global power consumption increased significantly, and robustness decreased in the same time. The inelastic shock occuring at foot landing spread into the upper part of the body, producing visible oscillations in the arms.

Experiments show that the walk of Acroban is robust, while remaining largely to be improved. Acroban is able to walk under various perturbations, including large one. It is able also to switch smoothly from horizontal ground to inclined ground (see video 6).

\section{EXPERIMENTS}

We set-up several kinds of tests on Acroban, such as putting it on a skateboard, walking on a flat or inclined soil or disrupting the balanced posture (e.g. in Video 4). Among these experiments, we selected the following ones in order to get quantitative measure of stabilisation process quality:

1) Evaluation of the postural balance of Acroban under controlled external perturbations.

2) Evaluation of balance when legs oscillate so that a dynamic weight transfer occurs.

These two experiments use the same compliant motor primitives controller to ensure the global balance of Acroban. Let us note that we achieved more quantitative experiments than what was possible to present within the space constraints of this paper.

\section{A. Evaluation of the Stabilization Process against External Perturbations}

For this experiment, we disturb Acroban with a controlled reproductible perturbation. This perturbation is generated by a pendulum composed with a weighted ball (see Fig.2.1.a.). In order to have a repeatable perturbation, we always drop the ball at the same altitude. The tracking camera is placed on the side of Acroban in order to get sagittal motion (see Fig.2.1.a.).

During 180 seconds, the robot receives twenty impacts. The phase diagram in the sagittal plane of the robot is shown on Fig.2.1.b. and the phase diagram of the principal displacement $X(t)$ is shown on Fig.2.1.c. Thanks to the motion capture system.
First, Fig.2.1.b. shows all the positions and speeds taken by the head of Acroban. This movement represents a closed system which here always converges towards equilibrium and return the neutral position $(0 ; 0)$ and zero velocity. Despite the disturbance, the robot is stable and here always finds its equilibrium converging to the neutral position.

Second, in order to quantify the convergence speed, one observes the phase diagram of the principal displacement $X(t)$. As shown on Fig.2.1.c. where each point represents the pairs $(X(t) ;(d X(t)) / d t)$, and each arrow represents the next state. It appears that the overall shape of this diagram is a spiral converging to the neutral position. The spiral reaches the neutral position after 6 or 7 laps.

So, Acroban oscillates up to seven times around its neutral position before finding its balance. This speed could certainly be improved, but it still shows a stable convergent dynamic behavior. This criterion is used to tune parameters of the motor primitive.

\section{B. Evaluation of the Stability of Dynamic Weight Transfer between Legs}

Here we evaluate the stability of Acroban's stabilization when legs oscillate causing a dynamic weight transfer from one leg to the other one. The tracking camera is placed in front of Acroban, we leave Acroban oscillate for 35 seconds and we record its motion in the coronal plane (see Fig.2.2.a).

First, we can then, as in previous sections IV-A, draw the phase diagram in the coronal plane. We obtain the Fig.2.2.b. This is a vector field whose points represent all the positions $(Y(t) ; Z(t))$ taken by the robot and the arrows represent the direction and value (gradient color and size) of the speed at the point. We see a closed curve on this diagram, which indicates that the motion is stable; the trajectory follows an eight. This diagram shows clearly the stable attractor dynamic and the typical "eight" shape found in the Human gait (see [33]).

Second, we can draw the phase diagram in the direction $Y(t)$. As shown in Fig.2.2.c., the phase forms a circle showing the stability of the trajectory: the robot never gets umbalanced nor deviates from its "orbit". Despite a low mechanical precision and a stabilization controller not optimised for this specific task (e.g. specific self-perturbation of the walking CPG), we have a controlled dynamics and a movement that is repeated in a deterministic and convergent way.

\section{Qualitative experiments}

Next to a number of quantitative experiments such as the one presented in the previous section, we also performed some qualitative experiments which results are illustrated in the videos accompanying this paper.

Postural stabilization. In addition to the study of the impact of deterministic perturbations on postural stabilization such as in previous section, we experimented a large variety of other potential large and diverste perturbations. As Video 4 shows, Acroban is able to maintain its balance when a real football ball (very big and rather heavy as compared to 

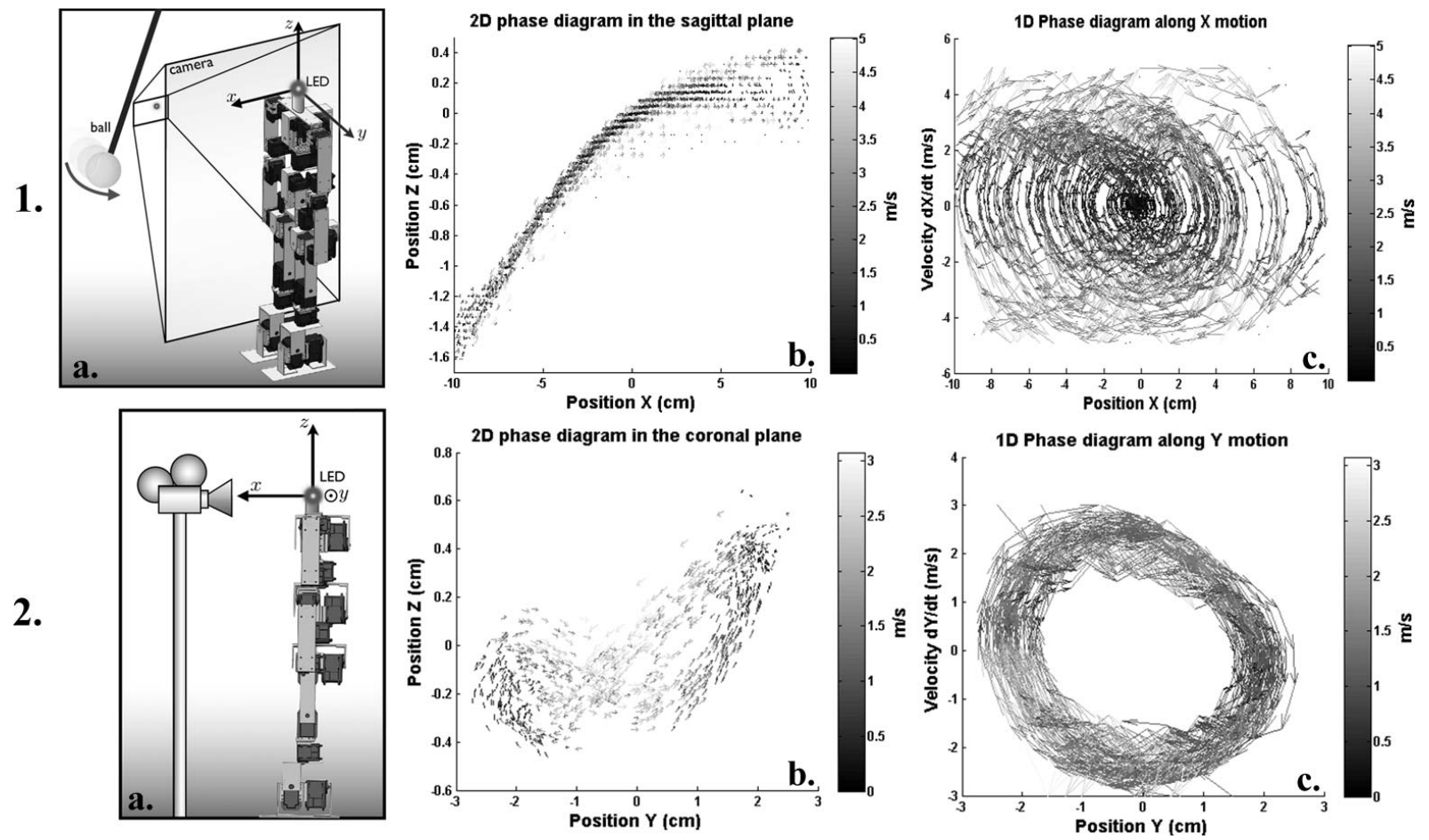

Fig. 2. 1.a.:Experimental 1 set up. We use a weighted ball $(154 \mathrm{gr})$ hanging from a rope $(87 \mathrm{~cm})$ for hitting Acroban in the torso in the direction of the vector $-x$. Before the drop, the ball is positioned so that the rope is parallel to the ground, so it hit Acroban with energy of 1.3J. 1.b.:This diagram shows the dynamic of the postural balance in the sagittal plane of Acroban under controlled external pertubations. It represents a closed system, converging to the neutral position, so the robot's behaviour is stable.1.c.: This diagram shows the phase diagram for the $X(t)$ motion of the dynamic of the postural balance of Acroban under controlled external pertubations. This diagram shows a converging spiral who needs up to seven laps to reaches the neutral position, showing a not very fast but still a stable and determined dynamic behavior. 2.a.:Experimental 2 set up.Acroban is placed in front of the camera. Data are measured in the plane $(\mathrm{y}, \mathrm{z})$ and Acroban has a CPG, doing an oscillation motion with his legs resulting in a rocking motion causing a dynamic weight transfer from one leg to each other.2.b.:This diagram shows the dynamic of the transfert of mass in the coronal plane. Points describe a kind of eight and this shape is closed and quite constant in the time, so the dynamic weight transfer is stable. 2.c.:This diagram shows the phase diagram for the $Y(t)$ motion of the dynamic mass transfert.This phase diagram is a circle, so the robot dynamic is stable.Despiste a low mechanical accuracy creating disturbances, the movement is constant, stable and determined.

its own size and weight) is thrown at him at non negligible speeds and at various places of its body as well as many impact angles. Furthermore, Acroban is able to maintain its postural balance when a human pushes or pulls many parts of its body. It is also possible to take Acroban by the shoulders, balance its whole body, and then literally throw it on the floor: if the throwing is reasonable, landing happens rather smoothly, the shock is absorbed by the structure compliance, and Acroban stays stood up. Finally, as shown at the end of Video 4, we even tried to launch Acroban on a skate-board, and this resulted again in quite robust ability to keep its balance.

Robustness to external perturbations while dynamically walking. As shown in Video 6, Acroban still keeps its balance when in addition a football ball is thrown at him while dynamically walking. The same holds when pushed by a human at various positions of its body (see also Video 1). When put on a skate-board pushed by a human, Acroban robustly oscillates its legs without falling. When put on a gentle downward or upward slope, Acroban continues to be able to go forward without falling.

Leading Acroban by the hand. An interesting behaviour, that we did not initially plan in the design of Acroban, happens when Acroban oscillates its legs with parameters such that it stays at the same position. As shown in Video 1 , when a human takes the hands of the robot in this configuration, like those of a little child, and try to lead him in a given direction, Acroban spontaneously follows. One can drive Acroban in any direction in such a way. This can actually be understood similarly to dynamic walking: the human applies a force on Acroban in a given direction, which makes it fall in that direction, but the robot continuously finds back its balance thanks to its postural stabilization.

\section{CONClusion And Future directions}

With its compliant structure and mechatronic, together with its bio-inspired vertebral column, and with a versatile stabilizing controller/motor primitive, Acroban can keep its balance in two major cases: postural stabilization and dynamic walking.

Future work will explore mainly three directions. First, while Acroban explores the role of of a complex vertebral colum and torso in semi-passive dynamic walking, the dynamics of its legs themselves could be largely improved, partly based on the use of modified CPGs as well as an optimized shape for the feet. The combination of its dynamic 
torso and whole body compliance with leg dynamics such as shown in [16], [7] will be of high interest. Second, the quantitative study of energy consumption of Acroban compliant dynamic walking should be pursued. Let us mention that Acroban has about one hour of autonomy with a 4-elements LiPo battery and using on-board computation. Third, as shown in Video 8, the fact that Acroban is lightweight, compliant and robust to external perturbations affords original physical human-robot interaction, even with children, which will be systematically explore.

\section{ACKNOWLEDGEMENTS}

We would like to thank Jérome Béchu, Adrien Baranes, Damian Cavaille, Loïc Gondry and Pierre Rouanet for discussions and technical help in this work. We also thank Jean-Charles Ceccato and Jean-René Cazalet for very helpful discussions. This research project was partially funded by ERC Starting Grant EXPLORERS 240007.

\section{REFERENCES}

[1] A. Albu-Schaffer, O. Eiberger, M. Fuchs, M. Grebenstein, S. Haddadin, C. Ott, A. Stemmer, T. Wimbock, S. Wolf, C. Borst, et al. Anthropomorphic Soft Robotics-from Torque Control to Variable Intrinsic Compliance. In 14th International Symposium on Robotics Research, 2009.

[2] C. Azevedo, P. Poignet, B. Espiau, B. Brogliato, D. Goeleven, J. Bourgeot, et al. Contrôle de la locomotion artificielle: de lhomme aux robots. In Second MIT Conference on Computational Fluid and Solid Mechanics, volume 9, pages 322-360, 2003.

[3] B. Bigge and I. Harvey. Humanoid Robots in Waseda UniversityHadaly-2 and WABIAN. Journal of Autonomous Robots, 12(1), 2002.

[4] J. Buchli and al. Compliant quadruped locomotion over rough terrain. Intelligent Robots and Systems. In IROS 2009. IEEE/RSJ International Conference on, pages 814-820, 2009.

[5] J. Ceccato. Le tronc, de la locomotion à la commande. $\mathrm{PhD}$ thesis, Universit de Montpellier2, 2009.

[6] D. Christensen, U. Schultz, and K. Stoy. A distributed strategy for gait adaptation in modular robots. In Robotics and Automation (ICRA), 2010 IEEE International Conference on, pages 2765-2770. IEEE, 2010.

[7] S. Collins, A. Ruina, R. Tedrake, and M. Wisse. Efficient bipedal robots based on passive-dynamic walkers. Science, 307(5712):1082, 2005.

[8] K. Daltorio, A. Horchler, S. Gorb, R. Ritzmann, and R. Quinn. A small wall-walking robot with compliant, adhesive feet. In Adhesive Feetâ, Intelligent Robots and Systems (IROS 2005), IEEE/RSJ International Conference on, 2005.

[9] V. Feipel, T. De Mesmaeker, P. Klein, and M. Rooze. Threedimensional kinematics of the lumbar spine during treadmill walking at different speeds. European Spine Journal, 10(1):16-22, 2001.

[10] M. Fuchs, C. Borst, P. Giordano, A. Baumann, E. Kraemer, J. Langwald, R. Gruber, N. Seitz, G. Plank, K. Kunze, et al. Rollin'Justindesign considerations and realization of a mobile platform for a humanoid upper body. In Proceedings of the 2009 IEEE international conference on Robotics and Automation, pages 1789-1795. Institute of Electrical and Electronics Engineers Inc., The, 2009.

[11] Y. Fukuoka, H. Kimura, and A. Cohen. Adaptive dynamic walking of a quadruped robot on irregular terrain based on biological concepts. The International Journal of Robotics Research, 22(3-4):187, 2003.

[12] F. Iida, J. Rummel, and A. Seyfarth. Bipedal walking and running with compliant legs. In 2007 IEEE International Conference on Robotics and Automation, pages 3970-3975, 2007.

[13] A. Ijspeert, A. Crespi, D. Ryczko, and J. Cabelguen. From swimming to walking with a salamander robot driven by a spinal cord model. Science, 315(5817):1416, 2007.

[14] S. Ito, H. Kawasaki, K. Moriki, and M. Sasaki. Robot Experiment of Torque Learning for Biped Balance with respect to Periodic External Force. In Advanced Robotics, 2005. ICAR '05. Proceedings., 12th International Conference on, pages 418-423, 2005.
[15] H. Marques, M. Jntsch, S. Wittmeier, C. Alessandro, O. Holland, C. Alessandro, A. Diamond, M. Lungarella, and K. R. Semipassive dynamic walking for humanoid robot using controllable spring stiffness on the ankle joint. In Proceedings of Humanoids2010, 2010.

[16] T. McGeer. Passive dynamic walking. The International Journal of Robotics Research, 9(2):62, 1990.

[17] G. Mennitto and M. Buehler. CARL: A compliant articulated robot leg for dynamic locomotion. Robotics and Autonomous Systems, 18(3):337-344, 1996.

[18] G. Metta, G. Sandini, D. Vernon, L. Natale, and F. Nori. The iCub humanoid robot: an open platform for research in embodied cognition. In Proceedings of the 8th Workshop on Performance Metrics for Intelligent Systems, pages 50-56. ACM, 2008.

[19] I. Mizuchi, T. Yoshikai, D. Sato, S. Yoshida, M. Inaba, and H. Inoue. Swing Motion by a Spined Whole-Body Tendon-Driven Humanoid" Kenta”. Nippon Robotto Gakkai Gakujutsu Koenkai Yokoshu (CDROM), 20:1C38, 2002.

[20] I. Mizuuchi, M. Inaba, K. Nagashima, R. Tajima, T. Yoshikai, Y. Kuniyoshi, and H. Inoue. Design and control of a flexible spine for the whole-body humanoid'Kenta'. Nippon Robotto Gakkai Gakujutsu Koenkai Yokoshu, 19:777-778, 2001.

[21] I. Mizuuchi, S. Yoshida, M. Inaba, and H. Inoue. The development and control of a flexible-spine for a human-form robot. Advanced Robotics, 17(2):179-196, 2003.

[22] K. Muecke and D. Hong. DARwIn evolution: development of a humanoid robot. In IEEE International Conference on Intelligent Robotics and Systems, 2007.

[23] A. Omer, R. Ghorbani, H. ok Lim, and A. Takanishi. Semi-passive dynamic walking for humanoid robot using controllable spring stiffness on the ankle joint. In int. conf. on Autonomous Robots and Agents (ICARA 2009), pages 681-685, 2009.

[24] J. Or and A. Takanishi. From lamprey to humanoid: the design and control of a flexible spine belly dancing humanoid robot with inspiration from biology. International Journal of Humanoid Robotics, 2(1):81, 2005.

[25] C. Paul. Morphological computation:: A basis for the analysis of morphology and control requirements. Robotics and Autonomous Systems, 54(8):619-630, 2006.

[26] C. Paul, M. Lungarella, and F. Iida. Morphology, control and passive dynamics. Robotics and Autonomous Systems, 54(8):617-618, 2006.

[27] S. Peter, S. Grimmer, S. Lipfert, and A. Seyfarth. Variable joint elasticities in running. Autonome Mobile Systeme 2009, pages 129136, 2009.

[28] R. Pfeifer, M. Lungarella, and F. Iida. Self-organization, embodiment, and biologically inspired robotics. Science, 318(5853):1088, 2007.

[29] M. Raibert, K. Blankespoor, G. Nelson, and R. Playter. Bigdog, the rough-terrain quaduped robot. Proceedings of the 17th International Federation of Automation Control.(April 2008), 2008.

[30] P. Rodman and H. McHenry. Bioenergetics and the origin of hominid bipedalism. American Journal of Physical Anthropology, 52(1):103106, 1980.

[31] J. Rose and J. Gamble. Human walking. Lippincott Williams \& Wilkins, 2006.

[32] U. Saranli, M. Buehler, and D. Koditschek. Design, modeling and preliminary control of a compliant hexapod robot. In IEEE International Conference on Robotics and Automation, volume 3, pages 2589-2596, 2000.

[33] J. Saunders et al. The major determinants in normal and pathological gait. The Journal of Bone and Joint Surgery, 35(3):543, 1953.

[34] U. Scarfogliero, C. Stefanini, and P. Dario. The use of compliant joints and elastic energy storage in bio-inspired legged robots. Mechanism and Machine Theory, 44(3):580-590, 2009.

[35] R. Tedrake, T. Zhang, M. Fong, and H. Seung. Actuating a simple 3D passive dynamic walker. In IEEE International Conference on Robotics and Automation, volume 5, pages 4656-4661, 2004.

[36] A. Thorstensson, H. Carlson, M. Zomlefer, and J. Nilsson. Lumbar back muscle activity in relation to trunk movements during locomotion in man. Acta Physiologica Scandinavica, 116(1):13-20, 1982.

[37] C. Vaughan. Theories of bipedal walking: an odyssey. Journal of biomechanics, 36(4):513-523, 2003.

[38] C. Walsh, K. Endo, and H. Herr. A quasi-passive leg exoskeleton for load-carrying augmentation. International Journal of Humanoid Robotics, 4(3):487-506, 2007. 